\title{
Intervención Psicoeducativa a un Alumno con Trastorno del Espectro Autista en Edad Tardía
}

\author{
Psychoeducational Intervention to a Student with Spectrum Autism \\ Disorder in Late Age
}

Noemí Vidal Castillo*

\section{RESUMEN}

El presente artículo describe la intervención realizada a un menor con autismo del Centro de Recursos para la Atención Integral al Espectro de Autismo (CRIAT). Usando la metodología de estudio de caso, se expone el proceso de atención psicoeducativa de un alumno de nivel preescolar, con el objetivo de mostrar los ajustes necesarios para la adaptación ambiental, curricular y en el proceso de inclusión de un alumno que recibió atención escolar por primera vez a la edad de 7 años. Los factores propios de este trastorno se agravaron por la atención tardía y por la ausencia de estimulación adecuada en su contexto educativo y familiar. La intención primordial de este estudio, es proponer estrategias para la adaptación e independencia que puedan servir como orientación para otros especialistas interesados en la materia.

Palabras clave: Autismo, estudio de casos, adecuación curricular, autonomía, educación especial, intervención psicoeducativa.

\section{ABSTRACT}

The following article describes the intervention made to an autism child from the Centro de Recursos para la Atención Integral al Espectro de Autismo (CRIAT in spanish). Using the case study methodology. The process expounds the psychoeducational attention of a preschool student with the purpose of to show the necessary adjustments for the ambient and curricular adaptation. Also the process of inclusión of a student that receives school attention at the age of 7 . The typical factors related to this disorder get worst due to an adequate stimulation in his educational and familiar context. The main intention of this study is to propose strategies for adaptation and independence that can serve as guidance for other specialists interested in the subject.
Keywords: Autism, Case studies, Curricular adaptation, Autonomy, Special education, Psychoeducational intervention

*mivica82@gmail.com

\section{INTRODUCCIÓN}

El autismo es un trastorno heterogéneo del neurodesarrollo con grados y manifestaciones muy variables que tiene causas tanto genéticas como ambientales (Morrison, 2015). La Organización Mundial de la Salud (OMS) (citada por Teletón.org s/f) estima que la incidencia del autismo a nivel mundial es de 1/160 nacimientos, sin embargo, mencionan que existen variaciones muy importantes entre las cifras de diversos estudios en el mundo.

En esta misma fuente señala que el Centers for Disease Control and Prevention (CDC por sus siglas en inglés), una de las instituciones de mayor prestigio en cuanto a datos y estadísticas, actualmente manejan la cifra de 1 caso de autismo por cada 68 nacimientos (Teletón, s.f.).

Las personas con Trastorno del Espectro Autista (TEA) presentan condiciones que se ven alteradas cualitativamente en el desarrollo social y comunicativo especialmente, y que permanecen a lo largo de toda la vida. Es importante destacar que no existen en la actualidad tratamientos que eliminen esta condición. Por tal motivo, la intervención temprana y la escolarización, son de suma importancia para estimular y favorecer una condición más óptima en el desarrollo de estos niños y jóvenes, potenciando su adaptación al medio social.

Bajo un contexto escolar, la enseñanza y aprendizaje de estos alumnos tiene que ser cuidadosamente organizada y planificada, proporcionando un entorno con una estructura que favorezca su independencia. 
Perspectivas Docentes 63

NTERVENCIÓN PSICOEDUCATIVA A UN ALUMNO CON TRASTORNO DEL ESPECTRO AUTISTA EN EDAD TARDÍA

PSYCHOEDUCATIONAL INTERVENTION TO A STUDENT WITH SPECTRUM AUTISM DISORDER IN LATE AGE

Noemí Vidal Castillo*

La escolarización de este alumnado se debe de brindar en ámbitos organizados dentro del contexto escolar, donde ellos desarrollen competencias que pueden favorecer su autonomía e inclusión en la vida social. Estos ámbitos organizados proporcionen propuestas adecuadas al continuo de necesidades psicoeducativas de los niños que presentan esta condición de vida.

El Centro de Recursos para la Atención Integral al Espectro de Autismo (CRIAT), ubicado en la ciudad de Villahermosa, Tabasco, es una institución a nivel estatal que atiende a niños y jóvenes con TEA, y tiene como objetivo, brindar atención integral a las personas con autismo, otorgando servicios que apoyen esta condición de vida y la de sus familias, a través de actividades asistenciales, educativas, recreativas, culturales, deportivas, así como su inclusión social, incluyendo actividades para desarrollar habilidades para la vida diaria, cognitivas, comunicativas, de aprendizaje funcional, y de sociabilización.

Este trabajo de investigación, se realizó en el CRIAT institución que ofrece apoyo inclusivo en el aprendizaje y la participación de la personas con esta condición de vida. Este estudio de caso, tiene el objetivo de describir las adecuaciones necesarias en la adaptación ambiental y curricular en el proceso de inclusión de un alumno con autismo de edad escolar, donde la condición propia del trastorno se agravan por la falta de atención y estimulación adecuada en un contexto educativo y familiar.

El CRIAT inicia sus labores en el año 2005 y durante este tiempo sus servicios se han diversificado, siendo así que en el 2015 se reorganiza este centro educativo trabajando en colaboración con el sistema estatal DIF y la Secretaria de Educación del estado.

\section{DESARROLLO}

Se describe a continuación, la intervención psicoeducativa realizada a un alumno al llegar a los servicios de atención escolarizada del CRIAT, teniendo como antecedente el no haber recibido con anterioridad ningún tipo de atención a pesar de estar en edad escolar. Cabe mencionar que el trabajo educativo oportuno es importante, porque cuando este no se brinda desde el momento en que se recibe el diagnóstico; su pronóstico de adaptación y desarrollo en todas las esferas de desarrollo y adaptación se ve comprometido.
EI CRIAT recibe alumnos con TEA con distintos niveles de afectación, sin embargo los niños que presentan mayores dificultades en su pronóstico de adaptación son aquellos que inician su atención psicoeducativa tardíamente. Por lo que la interrogación ¿Qué adaptaciones psicoeducativas se necesita implementar a un alumno con TEA que inicia su proceso escolar en una edad avanzada? Interrogante que permitió trazar el camino para delimitar acciones y estrategias para realizar la intervención.

Este estudio de caso se realizó con un alumno con autismo el cual presenta un retraso generalizado bastante significativo en su desarrollo. Para su atención implementamos la metodología del trabajo estructurado, agendas visuales, material concreto, atención personalizada y la orientación continua y permanente a los padres.

Al implementar esta intervención se buscó valorar las estrategias metodológicas adecuadas que permitieron favorecer los procesos adaptativos en el contexto escolar, además de promover la adaptación e inclusión al medio social de alumnos con TEA, desarrollando competencias de independencia, comunicativas y de socialización, a la vez que se brindó estrategias para el desarrollo de la calidad de vida del alumno de preescolar, que recibió atención por primera vez a la edad de 7 años.

\section{DISEÑO METODOLÓGICO}

Este estudio se abordó desde un paradigma cualitativo, ya que como sabemos este tipo de investigaciones tiene como eje principal el estudio de la realidad social tal y como esta se presenta cotidianamente; donde una de sus características principales es que es inductiva, se pueden desarrollar conceptos a partir de pautas de datos, y no desde hipótesis ya preconcebidas. Este diseño de investigación pretende ser flexible pues su punto de partida es el planteamiento a través de interrogantes. La problemática se abordó a través de un estudio de caso ya que este tiene como característica principal tratar de forma intensiva una unidad básica (Stake, 1999).

Esta investigación partió de un enfoque ideográfico realizando una descripción amplia y profunda de la intervención de un caso de un alumno con TEA, sin pretender partir de una hipótesis ni de generalizar las observaciones vertidas en la misma. Resaltando que los datos de este estudio procederán de la práctica y experiencia docente por consiguiente estos 
Perspectivas Docentes 63

NTERVENCIÓN PSICOEDUCATIVA A UN ALUMNO CON TRASTORNO DEL ESPECTRO AUTISTA EN EDAD TARDÍA

PSYCHOEDUCATIONAL INTERVENTION TO A STUDENT WITH SPECTRUM AUTISM DISORDER IN LATE AGE

Noemí Vidal Castillo*

serán basados ampliamente en la realidad, tratando de mostrar la complejidad del abordaje psicoeducativo de este alumno.

Teniendo como principales fuentes de información de este proceso de intervención a los especialistas del equipo multidisciplinario del CRIAT: las áreas de comunicación, computación, psicología, vida diaria, educación física, trabajo social y la intervención de la maestra de grupo, los cuales tuvieron participación con sus bitácoras de observación y los informes bimestrales.

Es importante mencionar que también fueron de gran utilidad para la descripción de este proceso de investigación el análisis de la evaluación psicopedagógica, el diario de clase, el cuaderno de notas, las guías de observación y la evaluación del desempeño y desarrollo del alumno. Organizando los datos de manera sistemática obtuvimos al final un informe del proceso integral de la intervención y los avances obtenidos en el desarrollo del alumno.

El concepto clásico de autismo en los últimos años se ha venido transformando, y en la actualidad se habla de un continuo. Para esta investigación se utilizó el que menciona el Manual diagnóstico y estadístico de las enfermedades mentales (DSM5), que describe como un trastorno del desarrollo neurológico con grados y manifestaciones muy variables que tienen causas tanto genéticas, biológicas y ambientales. El diagnóstico suele reconocerse en una fase temprana de la niñez, teniendo probabilidades altas y certeras aproximadamente a los 2 años y medio, persistiendo hasta la edad adulta; no obstante sus manifestaciones pueden modificarse en gran medida por su desarrollo, experiencia y la educación que se le brinde. Sus características se observan en tres categorías: problemas de comunicación, socialización y de conducta (Morrison, 2015).

Es necesario mencionar que la prevalencia de las personas con esta condición se ha visto incrementada a mediados de los años 90, como consecuencia de un mejor reconocimiento y el diagnóstico temprano, pudiendo reconocer que de cada 66 nacimientos 1 nace con TEA, (Cumi García, Guillén García, \& Zúñiga Carrasco, 2015) entendiendo entonces que al obtener un diagnóstico de TEA tiene que iniciarse una intervención oportuna en todas las esferas de su desarrollo.

A continuación se describen algunas señales de alarma que los padres pueden detectar en sus hijos y que les pueda indicar que es necesaria una evaluación por algún especialista.
- No observar sonrisas u otras expresiones placenteras a partir de los 6 meses.

- No responder a sonidos compartidos, sonrisas u otras expresiones a partir de los 6 meses.

- Falta de balbuceo.

- Ausencia de miradas hacia el la persona.

- No responder a gesto sociales como señalar, mostrar, decir adiós con la mano a partir de los 24 meses.

- Cualquier pérdida de lenguaje o habilidad social durante el desarrollo (Alcantud, Rico, \& Lozano, 2012).

Por consiguiente se ha demostrado que la intervención temprana intensiva puede mejorar el pronóstico de estos alumnos. Los programas de intervención aunque en sus diferentes enfoques y aproximaciones generalmente están orientados a potenciar las diferentes áreas del desarrollo del niño, fomentando una mayor independencia en la actividad diaria.

Hacemos a continuación una breve descripción de algunos modelos de intervención usados en la atención psicoeducativa de estos alumnos:

- Intervenciones Conductuales.- se basan en enseñar a los niños nuevos comportamientos y habilidades, usando técnicas especializadas estructuradas dentro de los que podemos mencionar método Lovas, ABA, Pivotal Response Training, Natural Languaje Paradigm, Incidental Teaching.

- Intervenciones Evolutivas.- se basan principalmente en el estudio del cambio de la conducta en relación con la edad, favoreciendo el desarrollo de los alumnos que no logran alcanzar el estándar normativo, tratando de encausar su potencial en aspectos cognitivos, psicomotrices y conductuales. Floor Time, Responsive Teaching, Relationship Development Intervention.

- Intervenciones Basadas en Terapias.-intervenciones centradas en la comunicación, estrategias visuales, instrucción con pistas visuales, lenguaje de signos, sistema de comunicación por intercambio 
Perspectivas Docentes 63

NTERVENCIÓN PSICOEDUCATIVA A UN ALUMNO CON TRASTORNO DEL ESPECTRO AUTISTA EN EDAD TARDÍA

PSYCHOEDUCATIONAL INTERVENTION TO A STUDENT WITH SPECTRUM AUTISM DISORDER IN LATE AGE

Noemí Vidal Castillo*

de imágenes, historias sociales (social stories), dispositivos generadores de lenguaje, comunicación facilitada, entrenamiento en comunicación funcional.

- Intervenciones Sensoriomotoras.- entrenamiento en integración auditiva, integración sensorial.

- Intervenciones Basadas en la Familia.- son aquellas que se orientan al trabajo con las familias, dando entrenamiento sobre las estrategias que se implementan en el contexto familiar para favorecer el desarrollo de sus hijos con TEA. Programas PBS (Family-Centred Positive Behaviour Support Programs), Programa Hanen (More than Words).

- Intervenciones Combinadas.- se basan en combinar los métodos evolutivos y conductuales. Modelo SCERTS, Modelo TEACCH, Modelo Denver, Modelo LEAP. (Mulas, y otros, 2010)

En el CRIAT la intervención está basada en la organización y la estructura de los espacios educativos con uso de claves visuales a través de pictogramas, adecuando el contexto del centro con la metodología TEACCH, favoreciendo el desarrollo de habilidades sociales y de comunicación, implementando un acompañamiento de orientación y guía a los padres con el fin de mejorar la calidad de vida y la convivencia en la familia.

Es importante mencionar que los alumnos con esta condición presentan dificultades en la anticipación y la contextualización pragmática del lenguaje por esta razón el ambiente estructurado y predecible se hace necesario en nuestro centro, ya que facilita el aprendizaje funcional a los niños, la estructura ambiental es un medio que favorece la flexibilidad mental y proporciona instrumentos que ayudan a prever, interpretar y manejar el futuro, en consecuencia los ambientes áulicos de nuestra escuela están organizados por áreas de trabajo en función del objetivo que se pretende, estimulando el desarrollo que le permitan autonomía en las actividades.

Describiendo los rincones o áreas de trabajo en que se divide la jornada escolar:

- El área verde o de transición: es el área donde los alumnos, realizan actividades desde su interés, como jugar, cantar, interactuar con sus pares, saludar, desayunar y despedirse; también se organizan actividades sociales que fomentan la espontaneidad y las actividades que muchas veces están fuera de una rutina o del orden establecido. Asimismo en esta área se organiza la agenda ya sea de forma concreta, de imágenes o escritas, trabajo de especial interés pues es el momento donde los niños organizan y estructuran las actividades que realizaran durante el día, es importante mencionar que las secuencias de las tareas debe ir señalada con claves visuales que faciliten la interpretación y el contexto donde se realizará tal actividad.

- El área amarilla o trabajo uno a uno: donde trabaja el docente con el alumno, los contenidos nuevos 0 aquellas actividades donde el alumno requiere más apoyo, esta área permite un contacto más directo con el alumno aquí se preparan actividades donde el niño va a ir desarrollando nuevas habilidades, el docente puede estar con el niño desde diferentes ángulos para favorecer la intervención (atrás, enfrente, a un costado) de acuerdo al objetivo al lograr.

- El área azul o individual es el área donde los alumnos trabajan independientemente con actividades que ellos conocen o manejan con más facilidad esta área brinda seguridad y satisfacción al poder realizar trabajos de manera autónoma, aunque también es importante mantener una actitud directiva por parte del docente y tener claro los objetivos, expectativas y procedimientos para facilitar el abordaje de la tarea por parte del alumno.

La rutina del día para todos los grados la podemos enumerar de la siguiente manera:

- Recepción de los alumnos y filtro de higiene y salud.

- Rutina física de $15 \mathrm{~min}$. en la explanada de la escuela donde participan todos los grupos, alumnos y maestros de la escuela.

- En el aula se inicia con el saludo con cantos y juegos simbólicos.

- Pase de lista con ayuda de los pictogramas.

- Realización de la agenda del día.

- Trabajo en mesa en las diferentes áreas.

- Trabajo de autonomía (lavado de manos, limpieza de mesa, colocación de mesa para el desayuno). 
Perspectivas Docentes 63

NTERVENCIÓN PSICOEDUCATIVA A UN ALUMNO CON TRASTORNO DEL ESPECTRO AUTISTA EN EDAD TARDÍA

PSYCHOEDUCATIONAL INTERVENTION TO A STUDENT WITH SPECTRUM AUTISM DISORDER IN LATE AGE

Noemí Vidal Castillo*

- Desayuno y hábitos alimenticios

- Lavado de manos y cepillado de dientes

- $\quad$ Actividades de juego y recreación en el receso.

- $\quad$ Actividades en el área de transición.

- Trabajo en las áreas.

- Habilidades de autonomía de cuidado y aseo personal

- Despedida nuevamente con cantos y juegos

La primera actividad es el eje rector del trabajo pues indica al alumno qué, cuándo, cómo y dónde se hacen las actividades que se realizan dentro del aula usando la agenda como guía que anticipa y contextualiza al alumno, esta agenda se usa a través de claves visuales, concretas o simbólicas apoyada en este caso de pictogramas.

Otra actividad importante que se promueve dentro de este centro son aquellas relacionadas con la independencia y autonomía personal pues estas elevan la calidad de vida de los alumnos y de la familia, así pues ir al baño, lavado de manos, la hora de los alimentos, el aseo y vestido personal cobran relevancia como parte de la curricula de nuestros alumnos.

La hora del juego también es una actividad que se promueve con especial interés haciendo uso de diferentes recursos lúdicos y aprovechando al máximo los espacios recreativos de la escuela, como el patio didáctico que ayuda a los alumnos a organizar sus actividades y mejorar sus habilidades sociales.

Otra intervención de vital importancia está relacionada con el desarrollo del juego simbólico donde se puede usar la música, el juego de roles, juego de turnos, actividades interactivas circulares como rondas etc.

La hora del trabajo en mesa es una de las actividades que se promueve para desarrollar nuevas habilidades y que constituyen la base para nuevos conocimientos apoyadas en la metodología Teacch, las claves visuales para las instrucciones y material concreto le dan al niño mayor entendimiento y funcionalidad a su quehacer cotidiano.

Con los más pequeños se desarrollan actividades de permanencia en su lugar, de atención, de competencias comunicativas y atención conjunta; son básicas para que puedan seguir evolucionando y desarrollando competencias que le permitan integrar y adquirir nuevas habilidades de autonomía.

Tenemos que decir que las actividades que se proponen toman en cuenta sus habilidades, pero de manera general en los grados de preescolar e intervención temprana las actividades son cortas y aumentan progresivamente en tiempo y dificultad de acuerdo a la evolución del pequeño.

Estas se presentan de manera clara y sencilla generalmente con instrucciones verbales y visuales; es importante que los niños comprendan el principio y el fin de cada una de ellas.

Durante el trabajo pedagógico, el docente tiene que hacer uso de diferentes estrategias que favorezcan las competencias del menor; podemos señalar dentro de éstas las siguientes:

- $\quad$ El uso de refuerzos positivos y estímulos constantes.

- El establecimiento de rutinas y reglas claras.

- $\quad$ Modelaje

- Uso de estrategias que favorezcan las conductas comunicativas.

- $\quad$ Actividades con materiales vistosos, manipulables y a los cuales se le puedan dar diferentes usos.

En el CRIAT, los criterios están basados en la relevancia, funcionalidad, adaptación del nivel evolutivo y la intervención ecológica, así pues estos estarán dirigidos a potenciar el máximo nivel de adaptación e independencia personal para que el alumno logre:

- Habilidades de interacción social

- La intervención en la atención conjunta.

- Las competencias básicas de autocontrol de la propia conducta.

- Estrategias de comunicación funcional a través de signos y pictogramas.

Estos objetivos generales de la intervención se fueron ajustando de acuerdo a las necesidades, características del niño y del entorno traduciéndose en objetivos más operativos, trabajando los siguientes contenidos: 
Perspectivas Docentes 63

NTERVENCIÓN PSICOEDUCATIVA A UN ALUMNO CON TRASTORNO DEL ESPECTRO AUTISTA EN EDAD TARDÍA

PSYCHOEDUCATIONAL INTERVENTION TO A STUDENT WITH SPECTRUM AUTISM DISORDER IN LATE AGE

Noemí Vidal Castillo*

\section{Autonomía}

- El cuerpo y sus necesidades básicas: alimentación, vestido y aseo.

- $\quad$ Diferentes actividades de la vida cotidiana.

- Orientación y desplazamiento.

\section{PROCEDIMIENTO}

Las actividades se desarrollaron en entornos naturales y estructurados dentro del centro educativo como el aula de clases, el área de independencia y de la vida diaria, y el patio, se asignaron tareas y responsabilidades como entrar a la escuela cargando su mochila y colocarla en su lugar, buscar lonchera, sentarse adecuadamente para desayunar, sacar y comer su desayuno, utilizar la cuchara o tenedor, limpiar su área de desayuno, guardar su lonchera, lavarse las manos y los dientes.

\section{Comunicación}

Uso de gestos y señas protodeclarativas, es decir gestos que utiliza el niño para compartir la atención del adulto respecto a un objeto o evento al que se señala.

Identificación de fotografías y pictogramas.

\section{PROCEDIMIENTO}

Es esta área uno de los ejes principales a trabajar para desarrollar los contenidos anteriores se basa en la anticipación de las rutinas a través de la lectura de la agenda mediante objetos, fotos y pictogramas y el desarrollo de la necesidad de comunicar a pedir ayuda a través de señas.

\section{Socialización}

El trabajo con la socialización en el alumno mencionado se basó en el desarrollo de los siguientes procesos.

Hábitos sociales: saludar, despedirse, compartir juguetes y el juego funcional, sonreír, tolerara a sus compañeros, tolerar el contacto físico.

\section{PROCEDIMIENTOS}

El trabajo se realizó primeramente con la anticipación y la predicción de tareas, la exploración del entorno, el ambiente escolar, la participación en eventos sociales y culturales del plantel, así como el uso de juego organizados previamente por el docente dando un papel al alumno guiándolo con apoyo total.

\section{DESCRIPCION DEL CASO}

Para el presente estudio se decide realizar una breve síntesis de los antecedentes e historia personal del alumno, resaltando aquellos datos que pueden ayudar a una mejor comprensión del trabajo realizado.

Seguido de esto se incluyen unas tablas de evaluación de las áreas que ilustran la situación en la que el niño llega por primera vez a la escuela y en la segunda tabla se muestra los progresos que se obtienen al finalizar el ciclo escolar de atención.

Para esta investigación tomamos un alumno de preescolar de 7 años de edad al que llamaremos Noé, alumno que llega a la escuela con una intervención psicoeducativa tardía, por lo tanto su desarrollo integral se ha visto frenado.

Noé fue planeado y deseado, durante la gestación la madre presentó ligeras altas de presión y en el tercer mes presentó síntomas de aborto, indicándole reposo absoluto; a las 35 semanas de gestación se decide adelantar el parto por vía cesárea debido a la falta de crecimiento del infante, por lo que su nacimiento fue prematuro, al nacer el menor no respiró por sí solo, y le dieron estimulación de bolseo. No requirió de incubadora.

Al nacer pesó $2.100 \mathrm{~kg}$ y midió $47 \mathrm{~cm}$, esto representa un peso inferior a lo esperado en el momento del nacimiento, su calificación de Apgar (examen clínico que se realiza al recién nacido después del parto para determinar cualquier necesidad inmediata de cuidados médicos) fue 7-9, lo cual es considerado un índice de desarrollo normal. El menor se alimentó de leche materna por medio de mamila complementando con leche de formula hasta los 7 meses, presentó una succión lenta tardando una hora para terminar una onza, el biberón lo utilizó hasta los 2 años aproximadamente, inició la ingesta de alimentos sólidos a los 5 meses, sostuvo su cabeza a los cinco meses, se sentó 
Perspectivas Docentes 63

NTERVENCIÓN PSICOEDUCATIVA A UN ALUMNO CON TRASTORNO DEL ESPECTRO AUTISTA EN EDAD TARDÍA

PSYCHOEDUCATIONAL INTERVENTION TO A STUDENT WITH SPECTRUM AUTISM DISORDER IN LATE AGE

Noemí Vidal Castillo*

a los once meses, gateó al año y dos meses y caminó con ayuda a los dos años. Todos estos indicadores del desarrollo son tardíos respecto a la tabla de desarrollo normal.

Noé forma parte de una familia compuesta y es el único hijo de ambos padres; sin embargo tiene un hermano mayor por parte de la madre.

El alumno es de complexión delgada, tez clara, cabello castaño, ojos claros, de talla y peso menor a su edad. Cuando Noé llega a la escuela sus habilidades cognitivas están por debajo del promedio de los niños de su edad, no existen antecedentes escolares anteriores y la madre informa que el niño hasta este momento está bajo el cuidado de una nana, que no tiene preparación en el área educativa, el tiempo que permanece bajo la tutela de dicha persona es de más de 10 horas al día porque ambos padres trabajan. El lugar donde pasa la mayor parte del tiempo el menor es un área de la casa restringida y donde está él solo con colchonetas y juguetes de los cuales no hacen un uso funcional.
A su llegada al centro educativo se observa un deterioro en su desarrollo cognitivo y un retraso psicomotriz significativo, ausencia de la comunicación expresiva con presencia de sonidos vocálicos, no hay conductas protodeclarativas, es decir, el alumno no comparte la experiencia ni tiene miradas con intención de comunicarse, ni protoimperativas, es decir, hay ausencias de gestos para solicitar ayuda. Se observa un deficiente control corporal dirigido a conductas funcionales habiendo presentes marchas sin sentido y como signo de protesta suele tirarse al suelo. Presenta nula motivación hacia las tareas escolares y dificultad en su proceso de adaptación al medio escolar.

\section{EVALUACION INICIAL}

Al finalizar el ciclo escolar se aplican nuevamente dichas baterías.

En la siguiente tabla enumeramos los instrumentos aplicados así como las áreas y procesos que se evalúan.

Tabla 1. CONCENTRADO DE EVALUACIONES

\begin{tabular}{|c|c|}
\hline INSTRUMENTOS APLICADOS & AREAS Y PROCESOS EVALUADOS \\
\hline $\begin{array}{l}\text { Guía valorativa curricular de competencias y habilidades para } \\
\text { niños con TEA (instrumento utilizado en el CRIAT). }\end{array}$ & $\begin{array}{l}\text { Rutina de trabajo. } \\
\text { Sistema de trabajo. } \\
\text { Imitación. } \\
\text { Socialización. } \\
\text { Lenguaje y comunicación. } \\
\text { Cognitivo/habilidades. } \\
\text { Comportamiento y adaptación. } \\
\text { Motricidad. }\end{array}$ \\
\hline $\begin{array}{l}\text { Formato de caracterización del alumno (adaptación del } \\
\text { inventario del espectro autista de Ángel Riviére). }\end{array}$ & $\begin{array}{l}\text { Trastorno cualitativos de la relación social. } \\
\text { Trastornos de las capacidades intersubjetivas y mentalistas. } \\
\text { Trastornos de las funciones comunicativas. } \\
\text { Trastornos cualitativos del lenguaje expresivo. } \\
\text { Trastornos cualitativos del lenguaje receptivo. } \\
\text { Trastornos de las competencias de anticipación. } \\
\text { Trastornos de la flexibilidad mental y comportamental. } \\
\text { Trastornos del sentido de la actividad propia. } \\
\text { Trastornos de la imaginación y de las capacidades de ficción. } \\
\text { Trastornos de la imitación. } \\
\text { Trastornos de la suspensión. }\end{array}$ \\
\hline
\end{tabular}

Fuente: Elaboración propia basada en los instrumentos de evaluación del CRIAT 2016. 
Perspectivas Docentes 63

NTERVENCIÓN PSICOEDUCATIVA A UN ALUMNO CON TRASTORNO DEL ESPECTRO AUTISTA EN EDAD TARDÍA

PSYCHOEDUCATIONAL INTERVENTION TO A STUDENT WITH SPECTRUM AUTISM DISORDER IN LATE AGE

Noemí Vidal Castillo*

En la siguiente tabla mostramos los resultados de la primera evaluación realizada a Noé, en la que se interpreta las condiciones en que fue recibido en el CRIAT.

Tabla 2. INTERPRETACIONES DE LAS AREAS EVALUADAS

\begin{tabular}{|c|c|c|}
\hline \multicolumn{3}{|l|}{ Nombre del alumno: Noé } \\
\hline \multirow{3}{*}{\multicolumn{3}{|c|}{$\begin{array}{l}\text { Edad: } 7 \text { años } \\
\text { Grado: } 3^{\circ} \text { de preescolar } \\
\text { Tipo de atención: escolarizada. }\end{array}$}} \\
\hline & & \\
\hline & & \\
\hline COMUNICACIÓN & SOCIALIZACIÓN & AUTONOMIA \\
\hline $\begin{array}{l}\text { Llanto continuo en ocasiones sin } \\
\text { sentido. } \\
\text { Presenta sonidos vocálicos pero sin } \\
\text { intención de comunicarse. } \\
\text { Suele tirarse al piso, bajarse el short } \\
\text { y quitarse los zapatos cuando se le } \\
\text { cierra la puerta del salón. } \\
\text { Presenta sialorrea, es decir, producción } \\
\text { excesiva de saliva. }\end{array}$ & $\begin{array}{l}\text { Acepta a proximidad del adulto por corto } \\
\text { tiempo. } \\
\text { Hipersensibilidad táctil, es decir, no tolera } \\
\text { el contacto físico como: abrazos, agarrarlo } \\
\text { de las manos o brazos, ni tocar su rostro. } \\
\text { No tiene un juguete de interés, y los que } \\
\text { logra agarrar no le da el uso funcional, } \\
\text { solo los tira. } \\
\text { Caminata sin sentido en el aula y patio de } \\
\text { la escuela. } \\
\text { Su permanencia sentado es de } 3 \\
\text { segundos máximo. } \\
\text { Falta de referencia conjunta. No hay } \\
\text { interés por las demás personas. }\end{array}$ & $\begin{array}{l}\text { Falta de control de esfínteres. } \\
\text { Se le guía con ayudad total para } \\
\text { dirigirse al salón. } \\
\text { Dificultad en cargar su mochila y } \\
\text { reconocer sus cosas. } \\
\text { En las habilidades básicas como la } \\
\text { alimentación, vestido y aseo se le } \\
\text { brinda el apoyo total. }\end{array}$ \\
\hline
\end{tabular}

Instrumentos de evaluación: Observación, guía valorativa curricular de competencias y habilidades para niños con TEA, informe de evaluación, cuaderno de evidencias.

Conclusión: el alumno presenta una desventaja notoria en cuanto a su desarrollo, especialmente en las áreas de comunicación, socialización e independencia, el cual se ven agravados por la falta de atención integral y la estimulación adecuada.

Se observa que los tipos de ayuda a las que responde, son la ayuda total, la instrucción verbal y las actividades de forma concreta.

Como resultado de las observaciones y evaluaciones se determina que las necesidades educativas especiales de Noé están relacionadas a los proceso de habilidades sociales, procesos perceptivos de permanencia en la tarea, habilidades motrices y de comunicación para lo cual se busca adecuar las propuestas de intervención psicoeducativas acorde a sus necesidades favoreciendo el desarrollo de sus competencias y la inclusión a la vida social.

Fuente: Elaboración propia. 
Perspectivas Docentes 63

NTERVENCIÓN PSICOEDUCATIVA A UN ALUMNO CON TRASTORNO DEL ESPECTRO AUTISTA EN EDAD TARDÍA

PSYCHOEDUCATIONAL INTERVENTION TO A STUDENT WITH SPECTRUM AUTISM DISORDER IN LATE AGE

Noemí Vidal Castillo*

En la siguiente tabla mostramos los resultados de la última evaluación realizada a Noé, en la que se interpreta las condiciones al término del ciclo escolar en el CRIAT.

Tabla 3. INTERPRETACIONES DE LAS AREAS EVALUADAS

\begin{tabular}{|c|c|c|}
\hline \multicolumn{3}{|l|}{$\begin{array}{l}\text { Nombre del alumno: Noé } \\
\text { Edad: } 7 \text { años } \\
\text { Grado: } 3^{\circ} \text { de preescolar } \\
\text { Tipo de atención: escolarizada. }\end{array}$} \\
\hline COMUNICACIÓN & SOCIALIZACIÓN & AUTONOMIA \\
\hline $\begin{array}{l}\text { Buscar al adulto y jalarlo de la mano } \\
\text { hacia dónde quiere ir o solicitar. } \\
\text { Mira esporádicamente con el adulto. } \\
\text { Atención visual hacia el objeto desea- } \\
\text { do. } \\
\text { Sonríe } \\
\text { Interpreta la agenda concreta, y sabe } \\
\text { qué actividad sigue. }\end{array}$ & $\begin{array}{l}\text { Tolera el contacto físico. } \\
\text { Acepta la presencia de sus compañeros } \\
\text { dentro y fuera del aula. } \\
\text { Disfruta estar en el patio. } \\
\text { Participa en eventos civicoculturales. } \\
\text { Conoce a las personas más cercanas a él. }\end{array}$ & $\begin{array}{l}\text { Permanencia sentado por lapsos de } \\
15 \text { minutos. } \\
\text { Tomar objetos pequeños con facili- } \\
\text { dad. } \\
\text { Desplazarse solo hasta su salón. } \\
\text { Conocimientos de sus pertenecías. } \\
\text { Cargar su mochila. } \\
\text { Realizar dos actividades con uso fun- } \\
\text { cional en mesa. } \\
\text { Sacar su termo, abrirlo y tomarlo. } \\
\text { Subir y bajarse del bricolin. }\end{array}$ \\
\hline
\end{tabular}

Fuente: Elaboración propia.

\section{RESULTADOS DE LA INTERVENCIÓN}

La intervención psicoeducativa con el menor en este proceso fue de 10 meses con un horario escolar de 8:00 a 12:30 am, respetando los ciclos vacacionales y los fines de semana; la asistencia del menor al centro educativa es constante y solamente se ha ausentado por motivos de salud en tres ocasiones durante este periodo.

La familia de Noé se muestra comprometida y responde favorablemente a las orientaciones ofrecidas para realizar el trabajo extraescolar en casa, estas recomendaciones están orientadas básicamente al trabajo de independencia personal y la inclusión del niño a ambientes sociales más normalizados.
Noé fue aceptando la rutina del trabajo poco a poco aunque al inicio no fue de su agrado pues cada vez que se le establecía la rutina él mostraba renuencias como llantos constantes, tirando los materiales, caminando sin sentido dentro y fuera del salón, tirándose al piso, quitándose los zapatos.

El programa que actualmente está establecido para él es de 3 minutos de trabajo por 5 minutos de descanso, mejorando su disposición corporal, y su disposición para el trabajo funcional; las actividades que se proponen siempre son con material concreto y con uso funcional para él.

Noé acepta el contacto físico de los adultos y gracias a ello se trabaja continuamente actividades que favorecen su referencia conjunta e imitación simples inducidas, éstas se hacen con ayuda total al alumno. 
Perspectivas Docentes 63

NTERVENCIÓN PSICOEDUCATIVA A UN ALUMNO CON TRASTORNO DEL ESPECTRO AUTISTA EN EDAD TARDÍA

PSYCHOEDUCATIONAL INTERVENTION TO A STUDENT WITH SPECTRUM AUTISM DISORDER IN LATE AGE

Noemí Vidal Castillo*

En el área de socialización el menor acepta la presencia de sus compañeros, disfruta la estancia en el patio y el juego al aire libre, sabe cuándo esta solo o cuando alguien está con él. Una forma de incitar la cercanía es que tira cosas, jala de la ropa y realiza sonidos guturales.

La manera de comunicarse de Noé evolucionó considerablemente, ahora él utiliza un lenguaje instrumental, toca y jala la mano, voltea a ver el objeto deseado, llora y hace sonidos vocálicos con la intención de satisfacer sus necesidades básicas.

Sus habilidades cognitivas da muestras de entender el entorno escolar, sabe cuándo se le solicita un trabajo, toma la plastilina y la amasa, mete y saca objetos grandes y pequeños de una bandeja, encima cuatro bloques grandes con ayuda total, pasa aros por un laberinto, sube y baja obstáculos, se dirige sin ayuda a sus pertenecías reconoce su mochila y el lugar que se le asigna; es capaz de desplazarse solo de la entrada de la escuela a su salón, participa en las actividades de educación física y de vida diaria con una mejor actitud. Sin embargo aún existe dificultad para seguir órdenes, resolver problemas y dificultad en la adaptación a las rutinas largas, se observa que Noé se siente perdido ante situaciones nuevas sin saber que hacer por lo que esta área tiene que seguirse estimulando a través de actividades organizadas y planificadas encaminadas a compensar y mejorar su estado actual.

\section{CONCLUSIONES}

Noé presenta una desventaja notoria en su desarrollo, especialmente en las áreas de comunicación y de independencia, las cuales se ven agravadas por la falta de atención integral y estimulación oportuna. Sin embargo, con la intervención adecuada y dirigida a compensar en la medida de lo posible las deficiencias, su desarrollo y adaptación al medio puede ir mejorando cada día, ya que el alumno logra responder favorablemente a los estímulos y a la intervención. Se observa que disfruta de la compañía del ambiente escolar y del uso de materiales didácticos, situación que genera condiciones para que él siga avanzando.

La experiencia del trabajo con este alumno ha sido ardua y es importante remarcar que no siempre fue fácil ya que al inicio la renuencia la falta de adaptación y de entendimiento al medio hacía que él tuviera desajustes emocionales y una respuesta negativa al trabajo. Pero, la constancia y el compromiso de la familia han sido factores que fungieron como un pivote que pueden seguir un camino de éxito y desarrollo mejorando la calidad de vida del menor y de la familia.

Es necesario enfatizar que cuando existe alguna condición de retraso o dificultad en cualquier área del desarrollo en un menor, la intervención entre más temprano se realice el pronóstico será más favorable.

\section{REFERENCIAS BIBLIOGRÁFICAS}

Alcantud, F., Rico, D., \& Lozano, L. (2012). Trastorno del espectro autista. Guía para padres y profesionales. Valencia: Universitat de Valencia.

Cumi García, C. A., Guillén García, L. M., \& Zúñiga Carrasco, I. R. (2015). El autismo en la actualidad. Boletín epidemiológico, $1-4$.

Felici, M. S. (2010). Intervenciones psicoeducativas en el espectro autista (tesis de maestría). Andalucía: Universidad Internacional de Andalucía.

Morrison, J. (2015). Guía para el diagnóstico clínico DSM-5. México: Manual Moderno.

Mulas, F., Ros-Cervera, G., Millá, M., Etchepareborda, M., Abad-Mas, L., \& Téllez de Meneses, M. (2010). Modelos de intervención en niños con autismo. Neurología, 50(3), 577-584.

Stake, R. E. (1999). Investigación con estudios de caso. Madrid: Morata.

Teletón. (s.f.). Teletón.org. Recuperado el 16 de Junio de 2016, de http://www.teleton.org/home/noticia/Que-es-el-Autismo 